Monatsschrift f. Geburtshülfe u. Gynäkologie 1926;72:I-IV

\title{
Contents, Vol. 72, 1926
}

Inhaltsverzeichnis

Origínalarbeiten. S $\quad$ Sit $\theta$

Baumui, Hans, liber Nachteile und Vorteile der Kiellandzange . 284 Broich, H. von, Die Bedeutung der Reinheitsgrade für den Ab-

lauf des Wochenbettes lit!)

Dienst, Arthur, Zur Frühdiagnose der Gravidität

Doerffer, Carl, Ein Fall von Phokomelie 195

Eufinger, Helnr., und Heimannsberg, L·., Adrenalin-Blut-

druckkurve und iScliwangerscliaftsvagotonJe 9

Graff, Erwin, Heilung einer puerperalen Blasenscheidentístel

durch Einpflanzung der Ureteren in den Darin . . 319

Heimannsberg, L., siehe Eufinger, Heinr.

Hinselmann, Hans, Beitrag zurKenntnis der Schädigungen der

unteren Genitalabschnitte bei Spontangeburten auf Grand syste-

matisoher kolposkopischer Untersuclaungen an Wöclmerinnen . 43 Hoppe, Gerhard, Eine

Pockenepidemie in einer Entbindu $\pi \mathrm{gs}-$

anstalt . 290

Istel, Franz, Über Inkarzeration des myomatösen Uterus . . . 315 Kamniker, 11., Klinik und

Atiologie der Deflexionslagen .... 129 Krukenberg, H., Zur Atiologie und Prophylaxe der angeborenen

Hüftverrenkung 295

Langen, B., Klinisches zur Adenouiyosis 303

Liegner, B., Quecksilbervergiftung von der Scheide aus 47

Luchs, K., Über Schwangerschaftsileus und verwandte Öymptomen-

komplexe $\quad 270$

Malfatti, Jos., Zur Frage des Schwangerschaftsileus 24

Martin, A., Zur Mutterschaftsptiege .. H6

Martin, Ed., Der Baräenheuersohe Schnitt 183

Montag, H., Über die Gefährlichkeit der manuellen Plazentarlösung

unter besonderer Berücksichtigung der Aortenkompression . . 150

Nettesheim, W., tlber Dysostosis cleidocranialis 159

Neuwirth, Karl, Abmahnende Erfahrungen gegentiber der sterili

sation jüngerer, zumal angeblich tuberkulöser Frauen

Oppenheim, Hans, $\Lambda \Gamma$ ersuche mit einem neuartigen Resorbens

(Ichthyol-Isapogen) 229

Pfalz, G. J., Vergleichende I⿳1/8tersuchungen zur Ermitthmg der

Streptokokken- und Staphylokokkenvirulenz298

Pup pel, Ernst, Beiträge zur Klinik der Pyelitis 224

Raaí'laub, W., Beitrag zur Kasuistik und Behandlung der Unfall- 
verletzungen der äußeren Genitalien, der Scheide und der

Abdominalwandungen 206

Rosenstein, Walter, .Zur Klinik und Histologie der Plazentar-

polypen $\cdot 264$

Scheffzek, F. A., Was geschieht zur Bekämpfung der Syphilis? 256 tíchmid, Hans Hermann,

Scheidenbildung aus der Harnblase . 330

IV Inhaltsverzeichnis.

Seile

8climitt, H. E., Zur Frage der Sektion und Pesorption des Erucht-

wassers und Hirer Störung bei Mißbildungen

1

Schneid er, Georg Heinrieh, Zur Differentialdiagnose chronischer

Adnexentzündung, subchronischer Appendizitis, Gallertkarzinoiu

des Wurmes und Pseudomyxoma peritonnaei

142

Schubert, G., und Steuding, Olga. Die Menstrualgiftfrage . 201 Stachow, Herbert. Erfahrungen über Clavipmin (Gehe) bei intra-

venöser Anwendung in der Geburtshilfe $\quad 58$

Steuding, Olga, sielie Schubert, G.

Theodor, Ludwig, Röntgenbehancllung akuter und subakuter

Entztindungen des weiblichen Geschlecbtsappara†es 09

Traugo It, $\mathrm{M}$ ar eel, Blutu $\mathrm{g}$ ex ovario oder Extra uterin-Gravidität $307 \mathrm{~W}$ a c li e $\mathrm{n} \mathrm{f} \mathrm{e} 1 \mathrm{~d} \mathrm{t}, 8$. v o n , Intrakardiale Adrenalininjektionen als Hilfs-

mittel bei Wiederbelebungsversuchen von sclieintoten Neu-

geborenen $\quad 1 \frac{1}{8}$

Weill, L., Zellulare Eutwicklungsmechanik. Nachtrag zu meinen

versehiedenen in dieser Monatssclirift erschienenen Arbeiten · 10 Zeitlin, L., Probepunktion des

hinteren Douglas bei Extrauterin-

gravidität $\quad 1 \mathrm{ti}<\mathrm{S}$

Zimmermann, ßol $>$., Zur Frage der Deziduabildung in der

graviden Tube $\cdot \mathrm{SO}$

Sauinielbericht. Die gynäkologische Strahlentherapie im Jahre 1924.

Von Dr. C. H. Engelbrecht-Erlangen HI

Hermann Periling $\uparrow$. Von Prof. S te id I - Baden-Baden L2(i

Alwin Mackeurodt $\uparrow 258$

Vereins- und Literaturbeilage.

Original-Siízungsberichte aus geburtshilflich-gynäkologischen Gesellschaften:

Mittelrlieinische Gesellschaft für Geburtshilfe und Gynäkologie.

66. Sitzung' am 28. VI. 1925 in Frankfort a. M ‘ 96

Mittelrlieinische Gesellschaft für Geburtshilfe und Gynäkologie.

67. Sitzung (25. X. 1925) in Frankfurt a. M. '....'.. 232

Niederrheinisch - westfälische Gesellscliaft für Geburtshilfe und

Gynäkologie. 75. Sitzung am 9. V. 1925356

Mitteldeutsche Gesellschaft für Geburtshilfe und Gynäkologie.

Sitzung am 8. XI. 1925 in Halle a. d. S 360

Münchener Gynäkologische Gesellschaft. Sitzung vom 2(i. II. 1925. 103 Gynäkologische

Gesellschaft in Breslau. Sitzung vom 25. X. 1925 . 337 Geburtshilflich-gvnäkolo < $\dot{i} \cdot$ ische

Gesellschaft in Wien. Sitzung vom 
10. XL 1925? .... ‘ ‘.. 242

Reîerate aus geburtshilflich-gynäk. Gesellschaften . . . 107, 243, 361

Geburtshîlflich-gynäkologísche Mîtteilungen aus anderen medi-

ziníschen Gesellschaften und Vereïnen .... 108, 245, 361

Buchbesprechungen 109, 247, 364

Literaturverzeichnis $117,252, \quad 368$

Personalien und Tagesnachrîchten 158,386 